\title{
Schilddrüse und Schwangerschaft: Schein und Sein
}

\section{Ein Streifzug durch die aktuelle Literatur im Licht der neuen Leitlinien}

\author{
Miriam Promintzer-Schifferl · Michael Krebs (D)
}

Eingegangen: 6. September 2018 / Angenommen: 19. Dezember 2018 / Online publiziert: 16. Januar 2019

(c) Der/die Autor(en) 2019

\begin{abstract}
Zusammenfassung Potentielle Funktionsstörungen der Schilddrüse treten bei bis $\mathrm{zu} 15 \%$ aller Schwangeren auf und gehören damit zu den häufigsten Fragestellungen, mit denen ÄrztInnen im Rahmen der Betreuung von Frauen mit Kinderwunsch/ Schwangerschaft in der klinischen Praxis konfrontiert sind. Die Grundzüge der neuen Empfehlungen der Fachgesellschaften und eine Auswahl der aktuellen wissenschaftlichen Literatur sollen hier diskutiert werden, um dem klinisch tätigen Arzt einen Überblick über neue Entwicklungen zu geben.
\end{abstract}

Schlüsselwörter Schilddrüse $\cdot$ Schwangerschaft $\cdot$ Hyperthyreose $\cdot$ Hypothyreose $\cdot$ Behandlung

\section{Thyroid disease in pregnancy}

Review of current literature and guidelines

Summary Impaired thyroid function is observed in $15 \%$ of all pregnancies and thus represents a relevant clinical issue. The key recommendations of currents as well as a selection of recently published literature are presented in this review.

Keywords Thyroid · Pregnancy · Hyperthyroidism • Hypothyroidism · Treatment

\section{Hypothyreose}

\section{Aktuelle Literatur}

Sind TSH-Konzentrationen im oberen Normalbereich mit ungeklärter Infertilität vergesellschaftet [1]?

Eine Infertilität unklarer Ursache besteht bei 10-30\% aller infertilen Paare. In dieser Untersuchung wurden TSH- und Prolaktin-Konzentrationen im Normalbereich im Serum von Frauen mit Infertilität unklarer Ursache $(n=187)$ mit gesunden Frauen verglichen, deren Partner an Azoospermie litt $(n=52)$.

Bei den Frauen mit Infertilität unklarer Ursache waren die TSH-Konzentrationen höher als in der Kontrollgruppe (TSH: 1,95 vs. $1,66 \mathrm{mU} / \mathrm{l}, p=0,003$ ). Doppelt so viele Frauen mit ungeklärter Infertilität hatten TSH-Werte über $2,5 \mathrm{mU} / 1$.

\section{Hat eine Behandlung mit Schilddrüsenhormon bei suboptimaler Schilddrüsenfunktion in der Schwangerschaft im langfristigen Verlauf einen Einfluss auf die Intelligenz der Kinder [2]?}

Eine ausreichende Versorgung mit Schilddrüsenhormon ist essentiell für die embryonale/fetale Hirnentwicklung. Da die kindliche Schilddrüse erst ab der 14.-18. Schwangerschaftswoche Hormone produziert, ist das fetale Hirn in der frühen Schwangerschaft völlig von der Versorgung durch die Mutter abhängig. Da die Auswirkungen einer moderaten Schilddrüsenfunktionsstörung der Mutter unklar sind, wurde in der CATS Studie untersucht, ob ein strukturiertes Screening und eine randomisierte Behandlung einen Effekt auf die kindliche Hirnfunktion haben. Fast 22.000 Frauen wurden um die 12. Schwangerschaftswoche gescreent. Bei niedrigem fT4 oder erhöhtem TSH wurde eine Behandlung mit $150 \mu \mathrm{g}$ T4 oder Placebo begonnen. Ein IQ Test im 3. Lebensjahr der Kinder hatte keinen Effekt der Therapie gezeigt. Aktuell wur- 
de die neurologische Entwicklung im 9. Lebensjahr der Kinder getestet und berichtet. Neben dem IQ wurden auch Gedächtnis und motorische Funktionen von Kindern 119 behandelter und 98 unbehandelter Frauen mit suboptimaler Schilddrüsenfunktion in der Schwangerschaft sowie von 232 Kindern von Frauen mit normaler Schilddrüsenfunktion untersucht.

Insgesamt fanden sich keine Unterschiede zwischen diesen Gruppen, sodass sich kein Hinweis auf einen positiven Effekt einer Behandlung mit Schilddrüsenhormon bei suboptimaler Schilddrüsenfunktion in der Schwangerschaft fand.

Ein wesentlicher Kritikpunkt im Studiendesign ist, dass ein Therapiebeginn in der 12.-13. Schwangerschaftswoche zu spät kommt, um die wichtige Hirnentwicklung in der Frühschwangerschaft zu fördern. Ein weiterer Kritikpunkt bezieht sich auf die hohe T4 Dosis, die zu einer Übertherapie mit potentiell negativem Effekt auf die Hirnentwicklung geführt haben könnte. Andererseits fand sich auch kein Unterschied zwischen dem IQ der Kinder von Frauen mit suboptimaler Schilddrüsenfunktion in der Schwangerschaft und Frauen mit normaler Schilddrüsenfunktion. Damit dürfte eine milde Schilddrüsenfunktionssstörung der Mutter in der Schwangerschaft keinen relevanten Effekt auf die Intelligenz der Kinder haben.

Führt die Therapie einer subklinischen Hypothyreose oder Hypothyroxinämie in der Schwangerschaft [3] zu einer besseren Intelligenz der Kinder?

Diese Studie untersuchte ebenfalls, ob eine Levothyroxintherapie bei schwangeren Frauen mit latenter Hypothyreose oder Hypothyroxinämie die kognitive Funktion ihrer Kinder verbessert. Die CATS Studie (Controlled Antenatal Thyroid Screening Study) hatte ja bereits 2012 gezeigt, dass die kognitive Funktion der 3-jährigen Kinder, deren Mütter aufgrund subklinischer Hypothyreose oder Hypothyroxinämie Levothyroxin erhielten, nicht besser war als die der Kontrollkinder. In der Studie von Casey et al. erhielten Kinder bis zum Alter von 5 Jahren jährliche Entwicklungs- und Verhaltenstests mit dem IQ als primären Endpunkt. Auch hier zeigte sich kein Unterschied im kognitiven Outcome zwischen Kindern, deren Müttern Levothyroxin erhielten oder nicht. In dieser Studie erfolgte der Einschluss noch später als in der CATS Studie, durchschnittlich in der 17. Schwangerschaftswoche. Das ist insofern ein bedeutender limitierender Faktor dieser Studie, da die fetale Schilddrüse bereits zwischen der 16. und 20. Schwangerschaftswoche ihre Funktion aufnimmt. Es gibt zwei weitere relevante Unterschiede zwischen dieser Studie und der CATS Studie. Erstens bestand in dieser Studie eine ausreichende Jodversorgung der Schwangeren (mittels Jodausscheidung im Harn bestimmt). Weiters wurden hier - im Gegensatz zu CATS - auch die TPO-Antikörper bestimmt, wobei eine Positivität keinen Effekt auf das Studienergebnis hatte.
Führt eine Thyroxintherapie von schwangeren Frauen mit latenter Hypothyreose ohne erhöhte Peroxidase Antikörper (TPO-AK) [4] zu einer Reduktion von geburtshilflichen Komplikationen?

In dieser Untersuchung wurden 366 schwangere Frauen mit normalen TPO-AK und latenter Hypothyreose (TSH >2,5 mU/l) mit 1092 euthyreoten schwangeren Frauen verglichen. Die Frauen mit erhöhten TSH wurden randomisiert mit $\mathrm{T} 4$ behandelt oder erhielten keine Schilddrüsenhormone.

Bei Frauen mit einem TSH-Wert >2,5 (ATA Leitlinie 2011) fand sich kein Unterschied in der Frühgeburtlichkeit zwischen der behandelten und der nicht-behandelten Gruppe. Jedoch in der Subgruppe der Frauen mit TSH >4 mU/l (entsprechend ATA Leitlinie 2017) war die Frühgeburtlichkeit in der behandelten Gruppe deutlich niedriger (RR: 0,38). Bei einem TSH-Grenzwert von $>4 \mathrm{mU} / 1$ war die "number needed to treat“ lediglich 3,8 um eine Frühgeburt zu verhindern! Diese Arbeit unterstützt somit den von der aktuellen Leitlinie propagierten höheren TSH-Grenzwert (siehe unten).

Gibt es eine Zusammenhang zwischen der mütterlichen Schilddrüsenfunktion in der

Frühschwangerschaft und den neurospychologische Leistungen der Kinder im 5. Lebensjahr [5]?

Im Rahmen einer Kohortenstudie wurden 1153 Frauen und ihre Kinder untersucht. Die Schilddrüsenfunktion der Mütter in der Frühschwangerschaft (TSH und fT4, um die 9. Schwangerschaftswoche) wurde aus eingefrorenen Serumproben bestimmt und die neuropsychologischen Leistungen der Kinder (Intelligenz, motorische Funktionen) wurden mit validierten Methoden gemessen.

Bei 145 Frauen (12,5\%) fand sich eine abnorme Schilddrüsenfunktion. Erhöhte TSH-Werte (>10 mU/l) und erniedrigte fT4 $(0,78 \mathrm{ng} / \mathrm{dl})$ Konzentrationen werden mit einem niedrigen verbalen IQ assoziiert. Bei Frauen mit TSH-Konzentrationen von $<0,1$ oder in den Bereichen von 2,5-5 mU/1 bzw. 5-9,99 mU/l fand sich kein Unterschied in der verbalen Intelligenz der Kinder verglichen mit euthyreoten Frauen (TSH $<2,5 \mathrm{mU} / \mathrm{l})$. Die Kinder von Müttern mit niedriger fT4 Konzentration zeigten eine schlechtere motorische Funktion sowie Verhaltensauffälligkeiten.

Während eine verminderte Intelligenz mit einer deutlichen Hypothyreose vergesellschaftet war, scheint für die Entwicklung der Motorik und der exekutiven Funktionen die T4 Konzentration wichtig zu sein. Diese Daten bestätigen die Studie von Haddow et al. [6], die zeigte, dass 48 Kinder von Müttern mit undiagnostizierter Hypothyreose (TSH 5-90 mU/l) im Alter von 7-9 Jahren einen leicht erniedrigten IQ hatten.

Hier war erst $a b$ einem TSH $>10 \mathrm{mU} / 1$ ein Einfluss auf den IQ nachweisbar, was die in der aktuellen Leitlinie empfohlene Anhebung des TSH-Grenzwerts in der Schwangerschaft unterstützt. Leider wurden die 
Schilddrüsenantikörper in dieser Studie nicht gemessen.

Interessanterweise fand sich eine nicht signifikante U-förmige Assoziation zwischen fT4 und kindlicher verbaler Intelligenz. Diese Daten erinnern an die Ergebnisse der "Generation R Study“, die einen Zusammenhang zwischen niedrigen und sehr hohen fT4 Konzentrationen der Mutter mit einem verminderten nichtverbalen IQ sowie einem reduzierten Volumen der grauen Substanz zeigten [7].

Haben subklinische Veränderungen der mütterlichen Schilddrüsenfunktion während der Schwangerschaft Auswirkungen auf das fetale Wachstum [8]?

Manifeste Schilddrüsenerkrankungen in der Schwangerschaft sind bekanntermaßen ein Risikofaktor für eine abnormale fetale Entwicklung und Wachstumsstörungen. Untersuchungen der Auswirkung milderer Schilddrüsenfunktionsstörungen auf das fetale Wachstum waren bisher weniger konklusiv. Diese Studie schloss 439 Schwangere ohne bekannte Schilddrüsenerkrankung ein und erhob in vier Visiten, die erste vor der 15. Schwangerschaftswoche, Schilddrüsenparameter (TSH, fT4, T3, T4) sowie fetale Maße wie Kopfumfang, Abdomenumfang, Femurlänge und geschätztes Gewicht sowie tatsächliches Geburtsgewicht. Es zeigte sich eine inverse Assoziation zwischen fT4 und Geburtsgewicht. Die stärkste Assoziation fand sich in der 10. Schwangerschaftswoche; zu diesem Zeitpunkt war ein um $10 \%$ erhöhtes fT4 mit einer Abnahme des Geburtsgewichts von 8,5g verbunden. Auch weitere biometrische Daten des Fetus wie intrauterines Wachstum, Bauch- und Kopfumfang waren negativ mit fT4 korreliert. Die Autoren diskutieren die Bedeutung des mütterlichen fT4 für das Wachstum der Weichteile als auch möglicherweise für das der Knochen. Eine supprimierte Deiodinaseaktivität der Plazenta könnte den Fetus höheren T4 und/oder T3 Konzentrationen aussetzen und dadurch Wachstum und Entwicklung beeinflussen.

Keine Assoziation hingegen zeigte sich in dieser Studie zwischen TSH und Geburtsgewicht oder Wachstumsmessungen. Vorliegende andere große Studien zeigten jedoch bei subklinischer Hypothyreose einen Zusammenhang mit kleinerem fetalen Kopfumfang und Körperlänge bei der Geburt [9] sowie eine intrauterine Wachstumsretardierung im dritten Trimester und ein niedriges Geburtsgewicht $(<2500 \mathrm{~g})$ [10].

\section{Diskussion und Überblick über die aktuelle Leitlinie der Amerikanischen Schilddrüsengesellschaft 2017}

Während in älteren Leitlinien ein generelles TSH-Ziel von $<2,5 \mathrm{mU} / \mathrm{l}$ empfohlen wurde, wird die Problematik in der aktuellen Empfehlung der amerikanischen Schilddrüsengesellschaft (ATA; [11]) deutlich differenzierter betrachtet und ein individuelles Vorgehen empfohlen, was die Situation für den Behandler leider nicht übersichtlicher macht.

Die zentrale Neuerung, basierend auf den Empfehlungen der aktuellen Leitlinien, besteht in der Einbeziehung des möglichen Vorliegens einer Autoimmunthyreoiditis in die Entscheidung, ob in der Schwangerschaft eine Substitutionstherapie mit Schilddrüsenhormon (T4) eingeleitet werden soll.

\section{Physiologie der Schilddrüsenfunktion in der Schwangerschaft}

Die um die 10. Schwangerschaftswoche maximale Freisetzung des Schwangerschaftshormons HCG führt, bedingt durch die (schwache) Bindung dieses Hormons an den TSH-Rezeptor, zu einer Stimulation der Schilddrüsenfunktion der Mutter. Dadurch sinkt im ersten Trimenon der Schwangerschaft die mütterliche TSH-Konzentration ab, während die Konzentration von freiem T4 passager leicht ansteigt. Rezente Studien haben gezeigt, dass dieser Mechanismus bei Frauen mit erhöhten TPO-Antikörpern als Marker einer bestehenden Autoimmunthyreoditis gestört ist (zusammengefasst in [12]). Erhöhte Schilddrüsen-Autoantikörper finden sich bei 2 bis $17 \%$ aller Schwangeren. Durch die verminderte funktionelle Reserve der Schilddrüse bei Autoimmunthyreoiditis kann der in der Schwangerschaft erhöhte Bedarf an Schilddrüsenhormon nicht immer gedeckt werden. Dadurch kann es trotz initial normalem TSH in ca. $20 \%$ zu einem Anstieg des TSH $>4 \mathrm{mU} / \mathrm{l}$ (latente Hypothyreose) kommen.

Für die klinische Praxis ergibt sich daher, dass bei euthyreoten Schwangeren mit erhöhten Schilddrüsenautoantikörpern regelmäßige Kontrollen der Schilddrüsenfunktion während der Schwangerschaft (initial alle 4 Wochen) empfohlen werden.

Wie oben erwähnt, sinkt die TSH-Konzentration in der Schwangerschaft normalerweise vor allem im ersten Trimenon etwas ab. Viele Leitlinien empfehlen daher bei Kinderwunsch und im ersten Trimenon der Schwangerschaft einen TSH-Zielwert von $<2,5 \mathrm{mIU} / \mathrm{L}$. Neuere Studien legen aber nahe, dass bei vielen verwendeten Assays und in vielen Populationen der obere TSH-Normalwert in der Schwangerschaft etwas höher liegen dürfte.

Die aktuelle Leitlinie der amerikanischen Schilddrüsengesellschaft empfiehlt daher das Erstellen von trimesterspezifischen Normalwerten auf Basis von Daten aus der lokalen Bevölkerung. Falls diese nicht verfügbar sein sollten, könnte vom oberen Normalwert für nicht-schwangere Erwachsene $0,5 \mathrm{mIU} / \mathrm{l} \mathrm{ab}$ gezogen werden. Dies entspricht in vielen Fällen einem Wert von ca. $3-4 \mathrm{mU} / \mathrm{l}$.

Aus den beiden genannten Neuerungen ergibt sich ein im Gegensatz zu den bisherigen Empfehlungen deutlich komplexeres Bild, das in der Abb. 1 zusammengefasst wird. Letztlich wird die Indikation zur Einleitung einer Thyroxintherapie in der Schwangerschaft durch Anwendung dieser Empfehlungen etwas 
Abb. 1 Behandlung einer Schilddrüsenfunktionsstörung in der Schwangerschaft. (Modifiziert nach [11]. *Siehe Erklärung im Text)

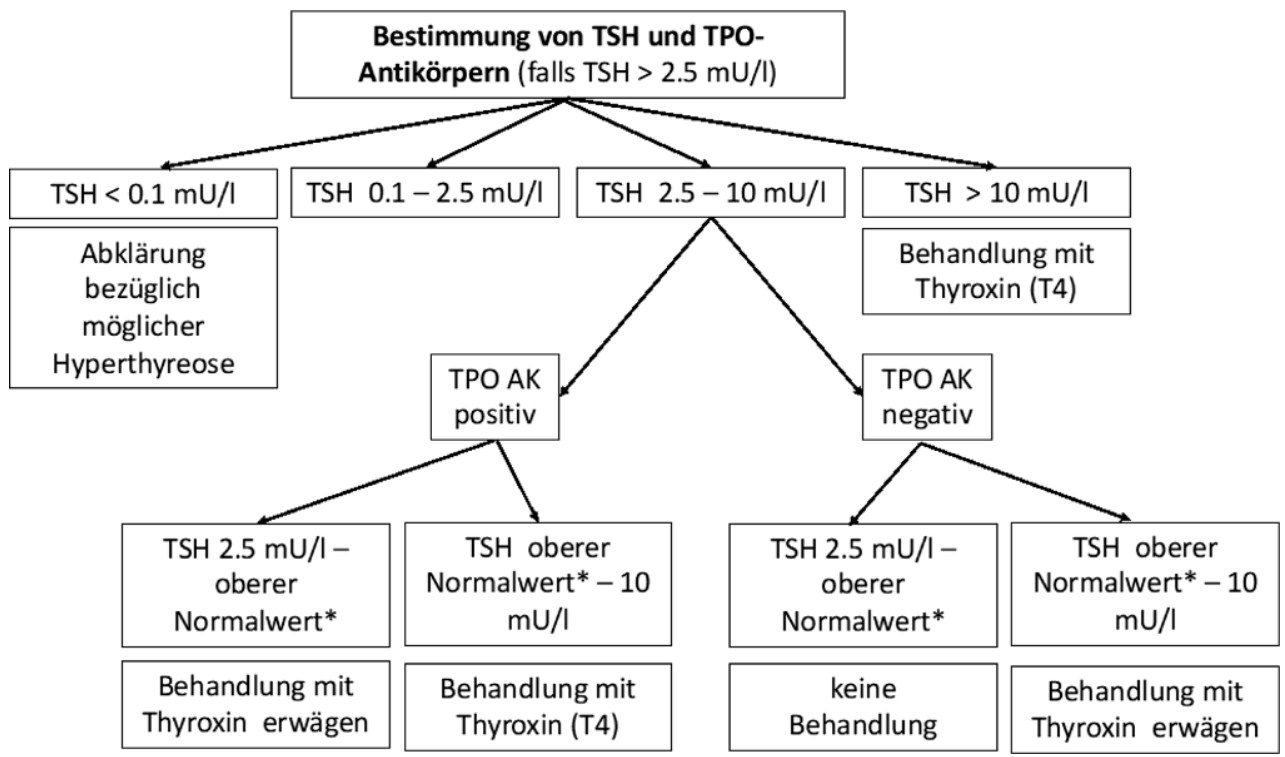

zurückhaltender gestellt werden. Neue Daten, die eine Assoziation nicht nur von erniedrigten, sondern auch erhöhten Konzentrationen von freiem T4 der Mütter mit einem minimal geringeren Intelligenzquotienten der Kinder zeigen, unterstützen möglicherweise diese Vorgangsweise oder zumindest eine vorsichtigere bedarfsgerechte Dosierung von Thyroxin [12].

\section{Hyperthyreose}

\section{Aktuelle Literatur}

Gibt es einen Zusammenhang zwischen der TSHRezeptor Antikörper (TRAK) Konzentration im mütterlichen Blut und dem Risiko für das Auftreten einer fetalen bzw. neonatalen Hyperthyreose [13]?

Morbus Basedow ist mit 0,1-0,2\% aller Schwangerschaften eine eher seltene Komplikation in der Schwangerschaft. TRAK sind üblicherweise im ersten Trimenon am höchsten und fallen meist gemeinsam mit einer Besserung der Hyperthyreose im Verlauf der Schwangerschaft ab. TRAK werden transplazentar in den Feten transportiert und können ab der ca. 20. Schwangerschaftswoche zu einer fetalen bzw. neonatalen Hyperthyreose führen. Bei allen Frauen mit M. Basedow in der Anamnese (auch nach definitiver Sanierung mittels Operation oder Radiojodtherapie) sollte daher in jedem Trimenon die TRAK Konzentration bestimmt werden.

In der hier vorgestellten Arbeit wurde im Rahmen einer Literaturstudie versucht, einen Grenzwert zu definieren, ab dem ein Risiko für eine Beeinträchtigung des Kindes besteht. Es fanden sich in der Literatur 53 Fallberichte einer fetalen bzw. neonatalen Hyperthyreose, wobei die niedrigste TRAK Konzentration, die mit einer Beeinträchtigung des Kindes assoziiert war, 4,4 U/l entsprechend 3,7-fach dem oberen Normalwert des Assays betrug.
Bei TRAK Konzentrationen $>3$-fach dem oberen Normalwert wird daher ein intensives geburtshilfliches Monitoring des Kindes empfohlen. Aufgrund der limitierten Evidenz für den Grenzwert sollten die TRAK Konzentrationen mit Vorsicht interpretiert werden.

\section{Führen thyreostatische Medikamente zu kongenitalen Malformationen [14]?}

Inadäquat oder unbehandelter Morbus Basedow kann zu Schwangerschaftsverlust oder maternalen und fetalen Komplikationen führen. Die medikamentöse Therapie stellt jedoch in mehrfacher Hinsicht eine Herausforderung dar. Die beiden zur Verfügung stehenden Thyreostatika, Propylthiouracil (PTU) und Methimazol (MMI), sind in der Schwangerschaft vergleichbar effektiv, passieren jedoch die Plazenta. In den vergangenen Jahren wurde aufgrund der seltenen, aber gut dokumentierten MMI-assoziierten Teratogenität PTU bevorzugt. Aufgrund der Sorge vor einer fatalen Hepatotoxizität unter PTU empfahlen die Guidelines PTU im ersten Trimester und dann einen Wechsel auf MMI.

Eine dänische Kohortenstudie berichtete 2013 über ein 1,4-fach erhöhtes Risiko von kongenitalen Malformationen bei Gabe von PTU im ersten Trimester [15]. Die aktuelle Studie ist eine sehr große Korea-weite Kohortenstudie mit 2.886.970 ausgetragenen Schwangerschaften und 12.891 Schwangerschaften, in denen die Frauen thyreostatische Medikamente im ersten Trimenon erhielten. Es bestätigte sich das erhöhte Risiko kongenitaler Malformationen bei Einnahme thyreostatischer Medikamente im ersten Trimenon. Für MMI stieg das relative Risiko für kongenitale Malformationen um 31 \% (entsprechend 17 zusätzlichen Fällen von Missbildung pro 1000 Lebendgeburten), für PTU um $16 \%$ (9 pro 1000 Lebendgeburten). Das erhöhte Risiko für MMI bestand auch fort, wenn Frauen 
bis zu 3 Monate vor der Schwangerschaft oder im ersten Trimenon auf PTU umgestellt wurden.

Wurde PTU bereits eingenommen, war ein Wechsel von PTU auf MMI mit einem höheren Fehlbildungsrisiko behaftet (um 47 Fälle pro 1000 Lebendgeburten) als wenn die Einnahme von PTU fortgesetzt wurde. Wird der Embryo zwei verschiedenen Teratogenen nacheinander ausgesetzt, scheint dies somit risikobehaftet zu sein! Der in den Leitlinien (siehe unten) empfohlene Wechsel von MMI auf PTU bei Eintritt einer Schwangerschaft wird durch diese Registerdaten nicht unterstützt.

Die Autoren empfehlen primär eine Diskussion mit den betroffenen Frauen hinsichtlich Familienplanung, effektiver Kontrazeption und definitiver Therapie. Als zweitbestes Vorgehen unterstützen die Autoren eine Medikamentenumstellung von MMI auf PTU vor Beendigung der Verhütung.

\section{Diskussion und Überblick über die aktuelle Leitlinie der Amerikanischen Schilddrüsengesellschaft (ATA) 2017}

Die aktuellen Leitlinien empfehlen, dass die Möglichkeit einer zukünftigen Schwangerschaft und das in dieser Situation komplexe Management der Erkrankung mit allen gebärfähigen Frauen besprochen werden soll. Bei Verdacht auf eine Schwangerschaft soll frühzeitig eine Bestätigung erfolgen und unmittelbar der betreuende Arzt aufgesucht werden.

Zu diesem Zeitpunkt soll ein Absetzen der thyreostatischen Medikation überlegt werden. Das kommt für Frauen in Frage, die unter einer niedrigen Dosis von MMI $(<5-10 \mathrm{mg} / \mathrm{d})$ euthyreot sind. Bei dieser Entscheidung sollen weiters die Dauer der Therapie, die Schilddrüsenmorphologie, die Erkrankungsdauer, der TRAK Titer sowie klinische Faktoren berücksichtigt werden. Schwangere, die eine thyreostatische Therapie benötigen, sollen in den ersten 16 Wochen PTU erhalten.

Entsprechend der ATA soll ein Umstieg von MMI auf PTU so früh wie möglich erfolgen (schwache Empfehlung, niedrige Evidenz).

Keine Empfehlung bei ungenügender Evidenz gibt die ATA in der Frage, ob nach den ersten 16 Wochen PTU fortgesetzt oder auf MMI gewechselt werden soll.

Bei Morbus Basedow in der Anamnese sollen, auch nach definitiver Therapie, TRAK in der Frühschwangerschaft bestimmt werden. Bei erhöhten TRAK in der Frühschwangerschaft soll eine Wiederholung der Bestimmung in der SSW 18-22 erfolgen sowie nochmals in der SSW 30-34. Zeigen sich auch in der Spätschwangerschaft erhöhte TRAK bzw. liegen diese $\mathrm{zu}$ irgendeinem Zeitpunkt der Schwangerschaft $>3$ fach über dem oberen Referenzbereich oder besteht eine unkontrollierte Hyperthyreose in der zweiten Schwangerschaftshälfte, ist ein fetales, neonatales und postneonatales Monitoring mit Bestimmung der
Herzfrequenz, Wachstum, Fruchtwassermenge und kindlicher Struma angezeigt.

Funding Open access funding provided by Medical University of Vienna.

Interessenkonflikt M. Promintzer-Schifferl und M. Krebs geben an, dass kein Interessenkonflikt besteht.

Open Access Dieser Artikel wird unter der Creative Commons Namensnennung 4.0 International Lizenz (http:// creativecommons.org/licenses/by/4.0/deed.de) veröffentlicht, welche die Nutzung, Vervielfältigung, Bearbeitung, Verbreitung und Wiedergabe in jeglichem Medium und Format erlaubt, sofern Sie den/die ursprünglichen Autor(en) und die Quelle ordnungsgemäß nennen, einen Link zur Creative Commons Lizenz beifügen und angeben, ob Änderungen vorgenommen wurden.

\section{Literatur}

1. Orouji Jokar T, Fourman LT, Lee H, Mentzinger K, Fazeli PK. Higher TSH levels within the normal range are associated with unexplained infertility. J Clin Endocrinol Metab. 2018;103(2):632-9.

2. Hales C, Taylor PN, Channon S, Paradice R, McEwan K, Zhang L, Gyedu M, Bakhsh A, Okosieme O, Muller I, et al. Controlled antenatal thyroid screening II: effect of treating maternal suboptimal thyroid function on child cognition. JClin Endocrinol Metab.2018;103(4):1583-91.

3. Casey BM, Thom EA, Peaceman AM, Varner MW, Sorokin Y, Hirtz DG, Reddy UM, Wapner RJ, Thorp JMJr., Saade G, et al. Treatment of subclinical hypothyroidism or hypothyroxinemia in pregnancy. NEngl J Med.2017;376(9):815-25.

4. Nazarpour S, Ramezani Tehrani F, Simbar M, Tohidi M, Minooee S, Rahmati M, Azizi F. Effects of levothyroxine on pregnant women with subclinical hypothyroidism, negative for thyroid peroxidase antibodies. J Clin Endocrinol Metab. 2018;103(3):926-35.

5. Andersen SL, Andersen S, Liew Z, Vestergaard P, Olsen J. Maternal thyroid function in early pregnancy and neuropsychological performance of the child at 5 years of age. JClin Endocrinol Metab. 2018;103(2):660-70.

6. Haddow JE, Palomaki GE, Allan WC, Williams JR, Knight GJ, Gagnon J, O'Heir CE, Mitchell ML, Hermos RJ, Waisbren SE, et al. Maternal thyroid deficiency during pregnancy and subsequent neuropsychological development of the child. NEngl J Med. 1999;341(8):549-55.

7. Korevaar TI, Muetzel R, Medici M, Chaker L, Jaddoe VW, de Rijke YB, Steegers EA, Visser TJ, White T, Tiemeier H, et al. Association of maternal thyroid function during early pregnancy with offspring IQ and brain morphology in childhood: a population-based prospective cohort study. Lancet Diabetes Endocrinol. 2016;4(1):35-43.

8. Johns LE, Ferguson KK, Cantonwine DE, Mukherjee B, Meeker JD, McElrath TF. Subclinical changes in maternal thyroid function parameters in pregnancy and fetal growth. JClin Endocrinol Metab. 2018;103(4):1349-58.

9. Su PY, Huang K, Hao JH, Xu YQ, Yan SQ, Li T, Xu YH, Tao FB. Maternal thyroid function in the first twenty weeks of pregnancy and subsequent fetal and infant development: a prospective population-based cohort study in China. J Clin Endocrinol Metab. 2011;96(10):3234-41.

10. Chen LM, Du WJ, Dai J, Zhang Q, Si GX, Yang H, Ye EL, Chen QS, Yu LC, Zhang C, et al. Effects of subclinical hypothyroidism on maternal and perinatal outcomes during 


\section{themenschwerpunkt}

pregnancy: a single-center cohort study of a Chinese population. PLoS ONE. 2014;9(10):e109364.

11. Alexander EK, Pearce EN, Brent GA, Brown RS, Chen $\mathrm{H}$, Dosiou C, Grobman WA, Laurberg P, Lazarus JH, Mandel SJ, et al. 2017 guidelines of the American Thyroid Association for the diagnosis and management of thyroid disease during pregnancy and the postpartum. Thyroid. 2017;27(3):315-89.

12. Korevaar TIM, Medici M, Visser TJ, Peeters RP. Thyroid disease in pregnancy: new insights in diagnosis and clinical management. Nat RevEndocrinol.2017;13(10):610-22.
13. van Dijk MM, Smits IH, Fliers E, Bisschop PH. Maternal thyrotropin receptor antibody concentration and the risk of fetal and neonatal thyrotoxicosis: a systematic review. Thyroid. 2018;28(2):257-64.

14. Seo GH, Kim TH, Chung JH. Antithyroid drugs and congenital malformations: a nationwide Korean cohort study. Ann Intern Med. 2018;168(6):405-13.

15. Andersen SL, Olsen J, Wu CS, Laurberg P. Birth defects after early pregnancy use of antithyroid drugs: a Danish nationwide study. JClin Endocrinol Metab. 2013;98(11):4373-81. 